\title{
Morfología de la caña de azúcar en la preparación profunda del suelo en canteros
}

\author{
Morphology of sugar cane in preparation and deep bed soil \\ Indiamara Marasca ${ }^{1 *}$, Reginaldo Barbosa Da Silva ${ }^{2}$, Maria Márcia Pereira Sartori ${ }^{1}$, \\ Antonio Paz Gonçalvez ${ }^{3}$, Kleber Pereira Lanças ${ }^{1}$
}

\begin{abstract}
RESUMEN
Las técnicas tradicionales de preparación de suelo han limitado la productividad de algunos cultivos. Facilitar el desarrollo de la planta mejorando mejorando las condiciones agrícolas, sin que ello afecte al ambiente, ha motivado la creación de nuevas tecnologías de producción en zonas rurales. Una de las tecnologías probadas es la preparación profunda de suelo, que promueve el desarrollo del sistema radicular de las plantas debido a una mejor aireación y baja compactación, lo que facilita la absorción de agua y nutrientes, ayudando a su desarrollo y favoreciendo una mayor productividad. Este trabajo tiene por objetivo evaluar el desarrollo morfológico de la planta en dos localidades de producción de cultivo de caña de azúcar, una de ellas manejada con maquinaria de preparación profunda de suelo en canteros y la otra, por los métodos de preparación convencional. El experimento fue realizado en Lençóis Paulista, São Paulo, en la empresa PHD, perteneciente al Grupo Zilor. Las variables analizadas fueron: macollamiento, diámetro del tallo, altura de planta, y su productividad. El macollamiento presentó mayores valores en la Preparación Profunda de Suelo en Canteros (PPC) en comparación con la Preparación Convencional (PC) a los 120 días de la siembra de caña de azúcar y después del corte y la altura de la planta únicamente presentó mayores valores para el PPC a los 390 días después de la siembra de caña de azúcar. Un ciclo (o corte) no fue suficiente para mostrar una diferencia significativa en la productividad de caña de azúcar entre los tratamientos analizados (Preparación Profunda de Suelo en Canteros y Preparación Convencional).
\end{abstract}

Palabras claves: preparación de suelos, labranza, subsolado, azada rotativa, Saccharum spp.

\begin{abstract}
The plant response referring to traditional techniques of soil management has limited the productivity of some crops. Allow the plant has a most conducive to its development without affecting the environment, has driven new technology in rural areas. With the deep tillage, the root system of the plants increases due to better aeration and reduced penetration resistance, facilitating the search for water and nutrients, helping in their development and ultimately providing greater productivity. This study aimed to evaluate the morphological development two areas of growing cane sugar one being managed with deep bed tillage equipment and other by conventional tillage methods. The experiment was conducted in Lençois Paulista, São Paulo the Company PHD Zilor belonging to the group. The variables analyzed were the tiller of the soil, the stem diameter, height of cane sugar and its productivity. The tillering showed higher values for the Preparation Deep Bed compared to conventional preparation at 120 days after planting of cane sugar and after cutting, and plant height only showed higher values for the Preparation Deep Bed to 390 days after planting of sugar cane. A cycle (or cut) was not enough to print difference in productivity of cane sugar by analyzed treatments (Deep Bed Tillage and Conventional Tillage).
\end{abstract}

Keywords: soil management, tillage, subsoiler, rotative row, Saccharum spp.

\section{Introducción}

Diversos factores pueden interferir en la productividad y en la calidad agronómica de la caña de azúcar que represente una integración de las diferentes condiciones del proceso de producción del cultivo (Gilbert et al., 2006).

Para la siembra de caña y nivelación del relieve del terreno los productores utilizan equipos de preparación intensiva y profunda del suelo que

1 Universidad Estatal Paulista "Julio de Mesquita Filho", Quinta Lageado Puerta I: Calle José Barbosa de Barros, № 1780. 18.610-307-Botucatu, SP. Teléfono: (14) 3880-7100. Botucatu, São Paulo, Brasil.

2 Profesor de la Escuela de Ingeniería Agronómica y Pesca. Universidad de São Paulo. Av. Nelson Brihi Badur, 430 Barrio: Vila Tupy, 11.900-000, SP. Brasil.

3 Facultad de Ciencias. Universidad de La Coruña. Campus A Zapateira, 15008, La Coruña, España.

* Autor para correspondencia: marasca@fca.unesp.br

Fecha de Recepción: 10 Septiembre, 2014.

Fecha de Aceptación: 25 Agosto, 2015. 
incluye la formación de canteros que se adaptan a una doble hilera de siembra, posibilitando una mayor infiltración de agua en el suelo. Sin embargo, necesitan de un trabajo de nivelación del relieve para conseguir mejores resultados cuando son combinados con el uso de piloto asistido (automático) en los tractores.

De acuerdo con Diola y Santos (2012), la fenología de la caña de azúcar se divide en cuatro fases de desarrollo: a) brotación y establecimiento del cultivo; b) macollamiento, entendiéndose como el periodo entre el final de la brotación hasta los 120 días después de plantada; c) desarrollo vegetativo y crecimiento de tallos, fase que se inicia inmediatamente después del macollamiento hasta los 270 días después de plantada; d) maduración, fase de síntesis y acumulación de azúcar, que dura de 270 a 360 días después de plantada.

Diola y Santos (2010) describen al macollamiento como un proceso fisiológico de ramificación subterránea continua de los nudos compactados al brote primario. Este proceso proporciona al cultivo el número de tallos necesarios para una buena producción. Macollos formados tempranamente ayudan a producir tallos más gruesos y más pesados, en tanto, que los formados tardíamente mueren o permanecen cortos o inmaduros. El número máximo de macollos se alcanza entre 90 y 120 días. A los 150-180 días, por lo menos el 50\% de estos mueren y el número de macollos se estabiliza.

La caña de azúcar se desarrolla en forma de matas, cuya parte aérea está formada por tallos, hojas, inflorescencias y semillas, mientras que la parte subterránea está compuesta por raíces y rizomas (Mozambani et al., 2006). La parte morfológica de la caña de azúcar de interés comercial es el tallo, que posee sacarosa industrializable. La composición química de los tallos es extremadamente variable en función de diversos factores como: la variedad del cultivo, edad fisiológica, condiciones climáticas durante el desarrollo y maduración; propiedades físicas, químicas y microbiológicas del suelo; y tipo de cultivo (Marques et al., 2001).

El tallo de caña de azúcar no presenta ramificaciones, su sección transversal es aproximadamente circular, diferenciado en segmentos compuestos por un nudo y un entrenudo. Los nudos son normalmente espaciados, en intervalos de 15 a $25 \mathrm{~cm}$, siendo más espaciados en la parte superior del tallo o vástago, donde se produce la elongación y, más próximos entre sí, en la base de la planta (Blackburn, 1984).
Las hojas son compuestas por vaina y lámina foliar y se unen al tallo en la base de los nudos, de modo alternado en dos líneas opuestas al vástago (James, 2003).

La anatomía y morfología de la caña de azúcar reafirma la capacidad especializada de la planta para acumular grandes cantidades de sacarosa. Al igual que otros miembros de la familia Poaceae, las partes de la planta por encima del suelo comprenden una serie de nudos que se encuentran separados por entrenudos en los que se desarrollan las yemas y las hojas, generados por un meristema apical vegetativo. Los entrenudos contienen las estructuras celulares especializadas en la transferencia y el almacenamiento de la sacarosa. Cuando se induce la floración, el patrón de desarrollo de los meristemas apicales se modifica transformándose en un gran raquis ramificado con numerosas flores bisexuales. Las raíces se producen ya sea como raíces de las plántulas después de la germinación de una semilla o como raíces adventicias originarias desde el nudo de la caña (Rae et al., 2014).

La caña de azúcar es una gramínea perenne, que macolla de manera abundante, en su fase inicial de desarrollo. Cuando se establece como cultivo, el autosombreado induce una inhibición del macollamiento y una aceleración del vástago principal. El crecimiento en altura continúa hasta la ocurrencia de alguna limitación o supresión de agua, de bajas temperaturas o debido al florecimiento, siendo este proceso indeseable en cultivos comerciales (Rodrigues, 1995).

Para el proceso de maduración, el cultivo de caña de azúcar exige temperaturas bajas o déficit hídrico, para que exista reposo fisiológico y mayor acumulación de sacarosa en los tallos (Andrade, 2006). En la región sureste del Brasil, este proceso sucede de forma natural a partir de abril/mayo finalizando en agosto (Deuber, 1988).

La caña de un año (12 meses), plantada en septiembre/octubre, tiene su máximo desarrollo entre noviembre y abril, disminuyendo después debido a las condiciones climáticas adversas del periodo lluvioso en el centro sur, pudiendo ser cosechada a partir de julio en función de la siembra (Rodrigues, 1995).

Genotipos de caña de azúcar responden a la altura de corte y a la época de cosecha de los tallos, con relación al macollamiento, y responden a la época de cosecha en relación con la productividad de los tallos. La reserva energética acumulada en la base de los tallos, por la altura del corte, favorece el rebrote de la caña de azúcar. La época de cosecha interfiere en la productividad de los tallos y de azúcar (Silva et al., 2008). 
Este trabajo tiene como objetivo evaluar el macollamiento, el diámetro del tallo, la altura de la caña de azúcar y su productividad en un área de caña de azúcar con ajuste al relieve del terreno, manejada con maquinaria de preparación profunda de suelo y también con manejo convencional.

\section{Materiales y Métodos}

El trabajo se realizó en la empresa PHD Cana, en el municipio de Lençóis Paulista - SP, en asociación con la Planta Zilor. El área del experimento se encuentra entre las coordenadas geográficas $22^{\circ} 40^{\prime} 30^{\prime \prime}$ Latitud Sur y $48^{\circ} 53^{\prime} 30^{\prime \prime}$ Longitud Oeste. El suelo del área experimental fue clasificado como Ultisol RojoAmarillo, textura arenosa media (EMBRAPA, 2013).

Para la caracterización química del suelo del área del experimento se colectaron cinco muestras simples de las capas $0-0,15,0,15-0,30$ y $0,30-0,45 \mathrm{~m}$, para formar una muestra compuesta de cada tratamiento con sus respectivos sistemas de preparación de suelo. Las muestras fueron colectadas al mismo tiempo que el muestreo de densidad y materia orgánica.

El experimento fue realizado en dos bloques, divididos en 4 franjas, con 4 repeticiones, para cada tipo de preparación, en los años 2012 y 2013, evaluando el efecto del equipo de Preparación Profunda del Suelo en Canteros (PPC), en relación con la Preparación Convencional (PC).

El comportamiento en función del tiempo de las variables de altura de la planta y diámetro del tallo fueron evaluadas por ajuste no lineal (función de Gompertz).

La siembra, en ambos tratamientos, fue realizada con espaciamiento entre hileras de plantas en el cantero de $0,90 \mathrm{~m}$ por $1,50 \mathrm{~m}$ y entre los centros de líneas de tránsito de 2,40 m (Figura 1).

Las plántulas utilizadas en la siembra del área experimental fueron de la variedad RB 966928, de 15 meses de edad, siendo su primer corte. Los análisis de morfología de las plantas fueron realizados siguiendo la metodología propuesta por Rodrigues (1995).

El macollamiento fue evaluado cuando el cultivo completó los cuatro meses, época en que el macollamiento se cierra según Rodrigues (1995), y después de cuatro meses de la cosecha. El conteo fue realizado en un espacio de 1,0 m dentro de cada ensayo, totalizando cuatro repeticiones por tratamiento.

El diámetro del tallo de caña de azúcar fue medido a la altura de $10 \mathrm{~cm}$, a partir del suelo. El crecimiento de la planta y el diámetro fueron evaluados cada tres meses. Las mediciones fueron realizadas a los $180,270,360$ y la cosecha a los 390 días, en cuatro plantas por tratamiento y por repetición.

Para el pesaje de tallos, paja y puntas de caña fue utilizada una célula de carga Líder en formato " $Z$ ", lector MK, control MK-2030, de cinco toneladas, como se muestra en la Figura 2.

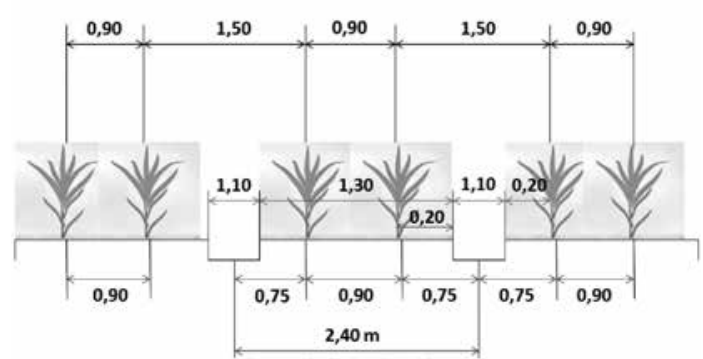

Figura 1. Espaciamientos utilizados para el cultivo de caña de azúcar en el experimento.

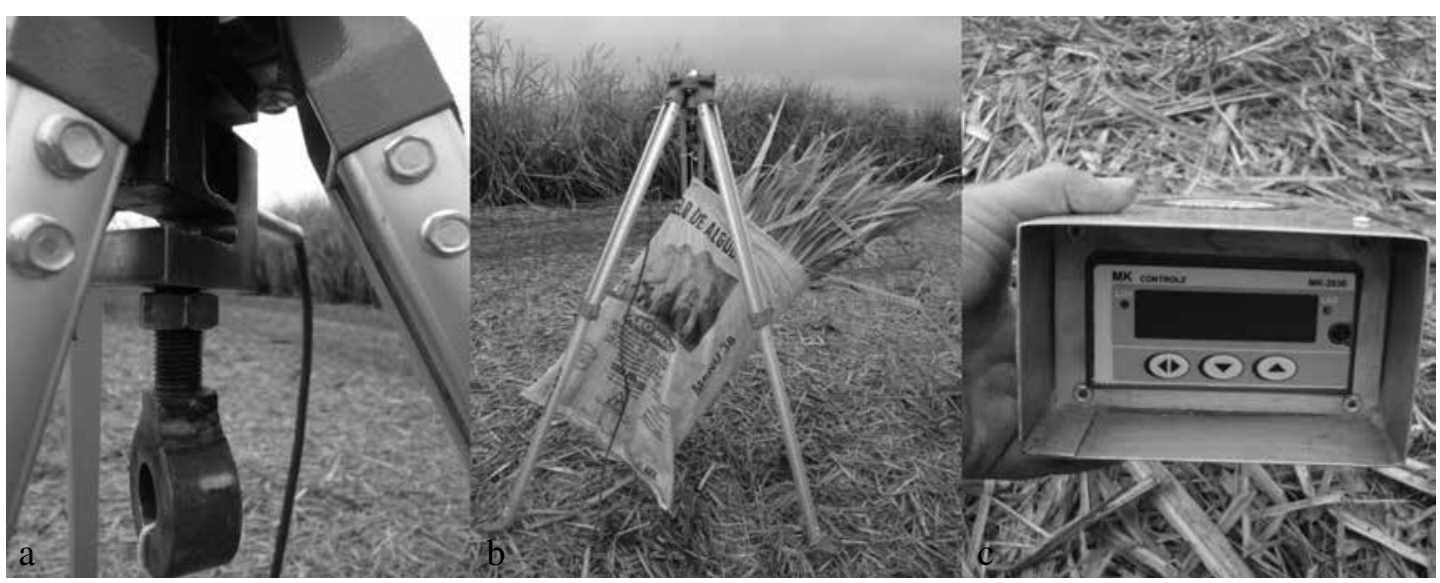

Figura 2. Célula de carga (a), trípode (b) y pantalla (c). 


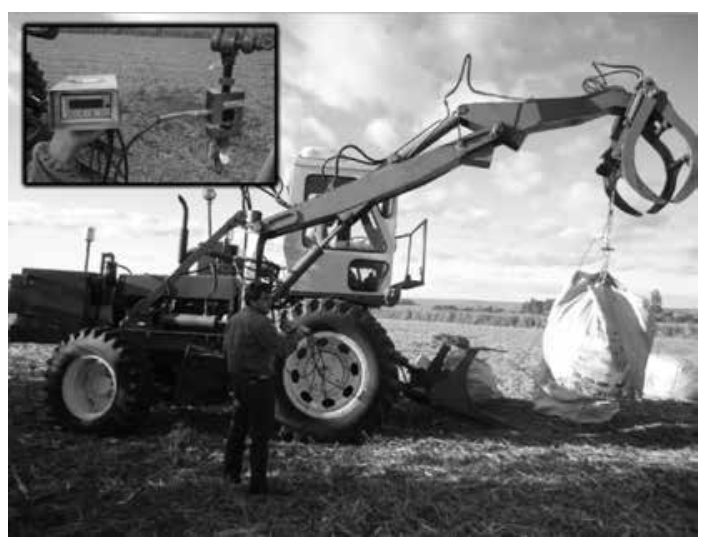

Figura 3. Equipo utilizado para el pesaje de los tallos y pajas.

La biometría del experimento fue realizada el día 14 de agosto de 2013. En el muestreo fueron evaluadas todas las plantas a lo largo de 10 metros de cada una de las hileras duales del cultivo, con cuatro repeticiones por tratamiento, total 80 metros de caña de azúcar muestreada. En esta evaluación fueron pesadas las siguientes partes de la planta: punta de caña, paja y tallo, separadamente. Como se muestra en la Figura 3 para paja y tallo.

\section{Resultados y Discusión}

En 2012 se pueden observar diferencias entre los valores de macollamiento dentro de los tratamientos, siendo PPC el que presenta una mayor cantidad de macollos. En 2013 no se observó la misma diferencia, no obstante, los valores de PPC fueron más homogéneos con relación a la PC (Figura 4).

La longevidad del cañaveral está directamente relacionada con la uniformidad de los macollos, para Morgado et al., (2000), la calidad de la plántula es fundamental, porque influye en el porcentaje de sobrevivencia, velocidad de crecimiento de los macollos y producción, las plántulas de mejor calidad evidencian un mayor potencial de crecimiento.

Los valores mostrados para macollamiento y altura en 2012 y 2013 presentan grandes variaciones para el tratamiento PC debido a la variabilidad del suelo.

La variabilidad del suelo también puede estar asociada con el comportamiento de la materia

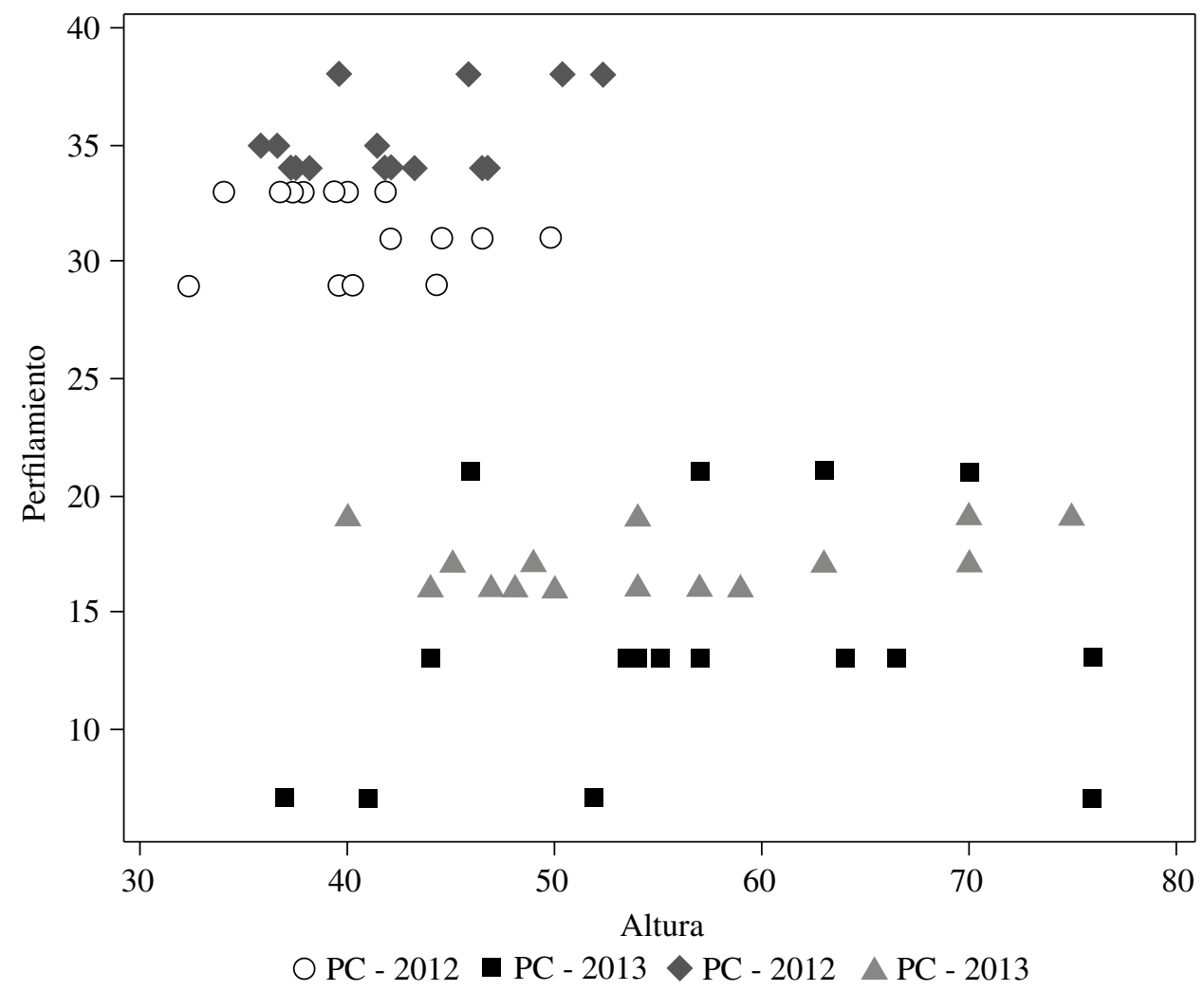

Figura 4. Diagrama de dispersión de macollamiento y altura de la planta a los 120 días en PPC - Preparación Profunda del Suelo y PC - Preparación Convencional en 2012 y 2013. 
orgánica. Santos et al. (2010) evaluó el crecimiento vegetativo de la productividad de caña de azúcar, en función del abono con bagazo de caña enriquecida con fósforo soluble (superfosfato triple), donde la aplicación de este material en la parte inferior del surco influye en el rendimiento de la caña y macollamiento.

El tratamiento PPC resultó en un número mayor de macollos en los Días Después de la Emergencia (DDE) y Días Después de la Cosecha (DDC). Las alturas de los macollos no presentaron diferencias significativas (Tabla 1 y 2 ).

El aumento del número de macollos puede estar asociado con la preparación profunda del suelo que posibilita un mejor desarrollo del cultivo en profundidad, haciendo que estos se mantengan estables en los periodos secos.

El diámetro del tallo no presentó diferencias entre los tratamientos para cada día de muestreo (Tabla 3). Los valores medios de altura de la planta a los 390 días tampoco mostraron diferencias estadísticas (Tabla 4), notándose un mayor aumento de altura de la planta en ese momento para el PPC.

La PPC presentó valores mayores que la PC después de 275 días, época donde se observa la respuesta de desarrollo del cultivo con relación al manejo (Figura 5).

El modelo de Gompertz se ajustó de mejor manera al comportamiento de crecimiento de caña de azúcar, según Fialho (1999), la función de Gompertz debe ser usada siempre que se analicen curvas de crecimiento tanto de animales como de tejido vegetal.

Los valores observados para estimar la productividad no presentaron diferencia estadística con la metodología utilizada, pero los $10 \mathrm{~m}$ de cada doble hilera evaluada ( $20 \mathrm{~m}$ en total) en el caso de PPC presentó $8 \mathrm{~kg}$ más de tallos que la PC, lo que en una hectárea serían $3.490 \mathrm{~kg}$ de diferencia (Tabla 5).

Los resultados de productividad encontrados en esta investigación (55.103 $\mathrm{kg} \mathrm{ha}^{-1}$ ) para la PPC fueron menores que la media registrada para el estado de São Paulo, (74.714 kg ha ${ }^{-1}$ safra 2012/2013), CONAB (2014).

Valores que no concuerdan con los de Galvani et al., (1997), donde comprobaron que la reducción del espaciamiento de $1,8 \mathrm{~m}$ a $0,9 \mathrm{~m}$ dio lugar a aumentos del $9 \%$ en la productividad agrícola, lo que representa una cantidad de 9,24 toneladas de caña por hectárea, valores superiores a los encontrados en este trabajo (tres toneladas).
Tabla 1. Valores medios del número de macollos y su altura a los 120 DDE (AÑO).

\begin{tabular}{lcc}
\hline Tratamientos & Macollamiento & Altura $(\mathrm{cm})$ \\
\hline PPC & $35 \mathrm{a}$ & $42 \mathrm{a}$ \\
PC & $31 \mathrm{~b}$ & $40 \mathrm{a}$ \\
\hline
\end{tabular}

Medias con una letra en común no difieren por la prueba de Tukey $(\alpha=0,10)$. DS: 5 ; CV: $2 \%$. PPC - Preparación Profunda del Suelo en Canteros y PC - Preparación Convencional.

Tabla 2. Valores medios del número de macollos y su altura a los 120 DAC (AÑ).

\begin{tabular}{lcc}
\hline Tratamientos & Macollamiento & Altura $(\mathrm{cm})$ \\
\hline PPC & $17 \mathrm{a}$ & $54 \mathrm{a}$ \\
PC & $13 \mathrm{~b}$ & $57 \mathrm{a}$ \\
\hline
\end{tabular}

Medias con una letra en común no difieren por la prueba de Tukey $(\alpha=0,10)$. DS: $10 ; \mathrm{CV}: 2 \%$. PPC - Preparación Profunda del Suelo en Canteros y PC - Preparación Convencional.

Tabla 3. Valores medios de diámetro del tallo.

\begin{tabular}{lcccc}
\hline \multicolumn{5}{c}{ Días } \\
\hline Tratamiento & 180 & 270 & 360 & 390 \\
PPC & $29,0 \mathrm{a}$ & 28,5 a & 29,5 a & 28,5 a \\
PC & $26,8 \mathrm{~b}$ & $28,1 \mathrm{a}$ & $28,2 \mathrm{~b}$ & $28,9 \mathrm{a}$ \\
\hline
\end{tabular}

Medias con una letra en común no difieren por la prueba de Tukey $(\alpha=0,10)$. DS: 0,6; CV: 0,02\%. PPC - Preparación Profunda del Suelo en Canteros y PC - Preparación Convencional.

Tabla 4. Valores medios de altura de las plantas evaluadas.

\begin{tabular}{lcccc}
\hline \multicolumn{5}{c}{ Días } \\
\hline Tratamiento & 180 & 270 & 360 & 390 \\
PPS & $1,62 \mathrm{a}$ & $2,70 \mathrm{a}$ & $3,00 \mathrm{a}$ & $3,40 \mathrm{a}$ \\
PC & $1,60 \mathrm{a}$ & $2,60 \mathrm{a}$ & $3,00 \mathrm{a}$ & $3,00 \mathrm{~b}$ \\
\hline
\end{tabular}

Medias con una letra en común no difieren por la prueba de Tukey $(\alpha=0,10)$. DS: 0,6; CV: $18 \%$. PPC - Preparación Profunda del Suelo en Canteros PPC y PC - Preparación Convencional.

Tabla 5. Valores medios de las variables utilizadas en la evaluación biométrica.

\begin{tabular}{lcc}
\hline & \multicolumn{2}{c}{ Tratamiento } \\
\hline Variables & PPC & PC \\
\hline Tallo $(\mathrm{kg}, 2$ líneas de 20 m en total) & $132 \mathrm{a}$ & $124 \mathrm{a}$ \\
Tallo $\left(\mathrm{kg} \mathrm{ha}{ }^{-1}\right)$ & 55104 & 51614 \\
Punta de caña $(\mathrm{kg}, 2$ líneas de 20 m en total) & $8 \mathrm{a}$ & $8 \mathrm{a}$ \\
Punta de caña $\left(\mathrm{kg} \mathrm{ha}^{-1}\right)$ & 3333 & 3333 \\
Paja $(\mathrm{kg}, 2$ líneas de 20 m en total) & $16 \mathrm{a}$ & $16 \mathrm{a}$ \\
Paja $\left(\mathrm{kg} \mathrm{ha}^{-1}\right)$ & 6667 & 6667 \\
\hline
\end{tabular}

Medias con una letra en común no difieren por la prueba de Tukey $(\alpha=0,10)$. DS: 7; CV: $13 \%$. PPC - Preparación Profunda del Suelo en Canteros y PC - Preparación Convencional. 


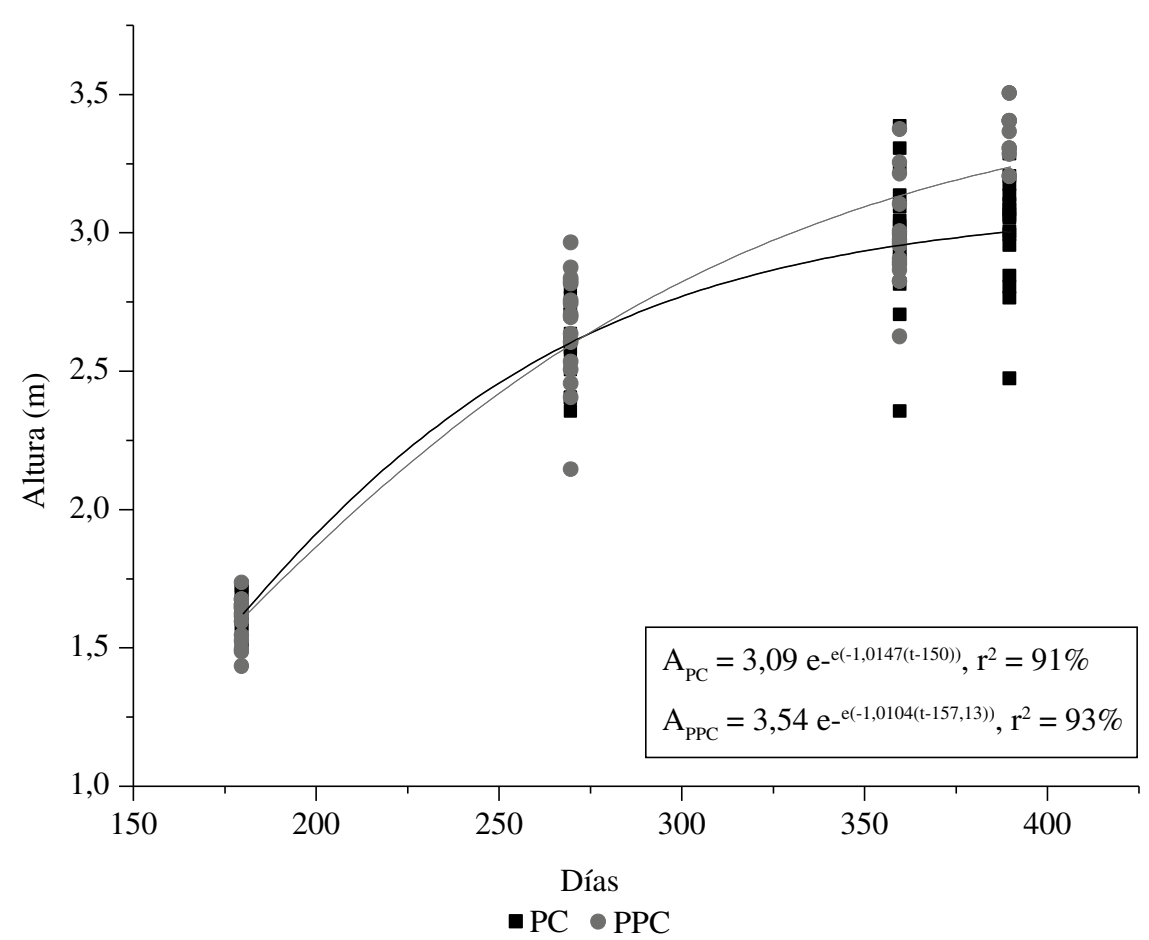

Figura 5. Comportamiento de la altura de la caña de azúcar en función de los días de finalización del macollamiento hasta la cosecha del cultivo.

A pesar de que la PPC presentó un aumento de productividad poco expresivo, es importante resaltar que el área del experimento fue irrigada con vinaza durante la sequía de julio, lo que puede haber influenciado sobre esta condición, evitando que la planta pase estrés hídrico, mostrando que el tratamiento se desarrolló mejor.

Para Reinert et al. (2008), la restricción del crecimiento de las raíces reduce de manera acentuada la productividad de los cultivos en años con déficit hídrico, siendo la PPC el tratamiento más productivo con relación a la PC en periodos de sequía, ya que una preparación profunda del suelo favorece un área de mayor movilidad en profundidad para el desarrollo de las raíces.

\section{Conclusiones}

El macollamiento presentó valores mayores para la Preparación Profunda de Suelo en Canteros (PPC) con relación a la Preparación Convencional (PC) a los 120 días de sembrada la caña de azúcar y después del corte y la altura de la planta únicamente presentó mayores valores para el PPC a los 390 días después de sembrada la caña de azúcar.

Un ciclo (o corte) no es suficiente para encontrar diferencias significativas en productividad de caña de azúcar para los tratamientos analizados (Preparación Profunda del Suelo en Canteros y Preparación Convencional).

\section{Literatura Citada}

Andrade, L.A. de B.

2006. Cultura da cana-de-açúcar. In: Cardoso, M. das G.

(Ed.). Produção de aguardente de cana-de-açúcar. 2a. ed. rev. e ampl. Lavras: UFLA, p. 25-67.

Blackburn, F.

1984. Sugar-cane. New York: Longman, 414 p.

CONAB - Companhia Nacional de Abastecimento da Safra Brasileira.
2014. Cana-de-açúcar. 2013/2014. Available in: http://www.conab.gov.br/OlalaCMS/uploads/ arquivos/13_08_08_09_39_29_boletim_cana_portugues__abril_2013_1o_lev.pdf Consulted: 07/ jan/2014).

Deuber, R.

1988. Maturação da cana-de-açúcar na região Sudeste do Brasil. In: IV Seminário de Tecnologia Agronômica, (1988, Piracicaba, BR) Anais... Piracicaba, BR: COPERSUCAR. p. 33-40. 
Diola, V.; Santos, F. Fisiologia. In: Santos, F.; Borém, A.; Caldas, C. (Eds.)

2010. Cana-de-açúcar: Bioenergia, açúcar e álcool - Tecnologias e perspectivas. Viçosa, $577 \mathrm{p}$.

Diola, V.; Santos, F. Fisiologia. In: Santos, F.; Borém, A.; Caldas, C. (Eds.)

2012. Cana-de-açúcar: Bioenergia, açúcar e álcool - Tecnologias e perspectivas. Viçosa: Os Editores, 2012. p. 25-49.

Diola, V.; Santos, F. Fisiologia. In: Santos, F.; Borém, A.; Caldas, C. (Eds.)

2010. Cana-de-açúcar: Bioenergia, açúcar e álcool - Tecnologias e perspectivas. Viçosa, $577 \mathrm{p}$.

EMBRAPA (Empresa Brasileira DE Pesquisa Agopecuária) Centro Nacional de Pesquisa de Solos.

2013. Sistema brasileiro de classificação de solos. $6^{\text {a }}$ Ed. Rio de Janeiro, $412 \mathrm{p}$.

Fialho, F.B.

1999. Interpretação da curva de crescimento de Gompertz. EMBRAPA - Centro Nacional de Pesquisa de Suínos e Aves. p. 1-4.

Gilbert, R.A.; Shine Junior, J.M.; Miller, J.D.; Rice, R.W.; Rainbolt, C.R.

2006. The effect of genotype, environment and time of harvest on sugarcane yields in Florida, USA. Field Crops Research, 95: 156-170.

James, G.

2003. Introduction to sugarcane. In: James, G. Sugarcane. Oxford: WileyBlackwell. p. 1-19.

Marques, M.O.; Marques, T.A.; Tasso Júnior, L.C.

2001. Tecnologia do açúcar. Produção e industrialização da cana-de-açúcar. Jaboticabal: Funep. p. 166.
Morgado, I.F.; Carneiro, J.G. DE A.; Leles, P.S.; Barros, D.G. 2000. Resíduos agroindustriais prensados como substrato para a produção de mudas de cana-de-açúcar. Scentia Agricola, Piracicaba, 57(4): 709-712.

Mozambani, A.E.; Pintos, A.S.; Segato, S.V. e Mattiuz, C.F.M. 2006. História e morfologia da cana-de-açúcar. In: Segato, S.V.; Pinto, A.S.; Jendiroba, E.; Nóbrega, J.C.M. Atualização em produção de cana-de-açúcar. Piracicaba: [s.n.], p. 11-18.

Rae, A.L.; Martinelli, A.P.; Dornelas, M.C.

2014. Anatomy and Morphology, In: Moore, P.H.; Botha. F.C. Sugarcane: physiology, biochemistry, and functional biology. 1 ed.: John Wiley \& Sons Ltd. pp. 19-34.

Reinert, D.J. Albuquerque, J.A.; Reichert, J.M.; Aita, C.; Andrada, M.M.C. 2008. Limites críticos de densidade do solo para o crescimento de raízes de plantas de cobertura em Argissolo Vermelho. Revista Brasileira de Ciência do Solo, 32: 1805-1816.

Rodrigues, D.J.

1995. Fisiologia da Cana-de-Açúcar. Universidade Estadual Paulista, Instituto de Biociências, Campus de Botucatu. Botucatu, p. 101.

Santos, D.H.; Tiritan, C.S.; Foloni, J.S.S.; Fabris, L.B.

2010. Produtividade de cana-de-açúcar sob adubação com torta de filtro enriquecida com fosfato solúvel. Pesquisa Agropecuária Tropical, 40(4): 454-461.

Silva, M.A.; Jeronimo, E.M., Lúcio, A.D. 2008. Perfilhamento e produtividade de cana-de-açúcar com diferentes alturas de corte e épocas de colheita. Pesq. agropec. bras., 43(8): 979-986. 
